\title{
Pharmacokinetics and Exposure-Response Relationship of Teprotumumab, an Insulin-Like Growth Factor-1 Receptor-Blocking Antibody, in Thyroid Eye Disease
}

\author{
Yan Xin ${ }^{1} \cdot$ Fengyan X $\mathbf{u}^{2} \cdot$ Yuying Gao ${ }^{2} \cdot$ Nivedita Bhatt $^{1} \cdot$ Jason Chamberlain ${ }^{1} \cdot$ Saba Sile $^{1} \cdot$ Suzy Hammel $^{1}$. \\ Robert J. Holt ${ }^{1}$ - Srini Ramanathan ${ }^{1}$
}

Accepted: 10 February 2021 / Published online: 26 March 2021

(c) The Author(s) 2021

\begin{abstract}
Background and Objective Thyroid eye disease (TED) is characterized by inflammation/expansion of orbital tissues, proptosis, and diplopia. Teprotumumab is the first US Food and Drug Administration-approved therapy for TED, administered as an initial intravenous infusion of $10 \mathrm{mg} / \mathrm{kg}$ followed by $20 \mathrm{mg} / \mathrm{kg}$ every 3 weeks for an additional seven infusions. The objective of this article is to discuss the pharmacokinetics and exposure-response profile for teprotumumab in patients with TED. Methods A population pharmacokinetic analysis was performed to characterize pharmacokinetics and select dosing in patients with TED. Exposure-response was evaluated for efficacy (proptosis response, clinical activity score categorical response, and diplopia response) and safety (hyperglycemia, muscle spasms, and hearing impairment) parameters.

Results Teprotumumab pharmacokinetics was linear in patients with TED, with low systemic clearance ( $0.334 \mathrm{~L} /$ day), low volume of distribution (3.9 and 4.2 L for the central and peripheral compartment, respectively), and a long elimination halflife (19.9 days). The approved dosing regimen provided $>20 \mu \mathrm{g} / \mathrm{mL}$ for $>90 \%$ insulin-like growth factor 1 receptor saturation throughout the dosing interval. Model-predicted mean ( \pm standard deviation) steady-state area under the concentration-time curve, peak, and trough concentrations in patients with TED were $131( \pm 30.9) \mathrm{mg} \cdot \mathrm{h} / \mathrm{mL}, 643( \pm 130) \mu \mathrm{g} / \mathrm{mL}$, and $157( \pm$ 50.6) $\mu \mathrm{g} / \mathrm{mL}$, respectively. Female patients had a $15 \%$ higher steady-state peak concentration but a similar steady-state area under the concentration-time curve vs male patients. No other covariates affected teprotumumab pharmacokinetics. No meaningful correlations between teprotumumab exposures and efficacy or safety parameters were observed.

Conclusions Teprotumumab pharmacokinetics was well characterized in patients with TED, and generally consistent with other IgG1 antibodies. Efficacy was consistent across the exposure range with a well-tolerated safety profile supporting the current dose regimen for patients with TED.
\end{abstract}

Yan Xin

yxin@horizontherapeutics.com

1 Horizon Therapeutics plc, 150 S. Saunders Rd, Lake Forest, IL 60045, USA

2 Shanghai Qiangshi Information Technology Co., Ltd, Shanghai, China

\section{Key Points}

The approved teprotumumab dose regimen for patients with thyroid eye disease was supported by consistent efficacy across exposure ranges with a well-tolerated safety profile.

Teprotumumab pharmacokinetics in patients with thyroid eye disease was well characterized and generally consistent with other IgG1 antibodies. 


\section{Introduction}

Thyroid eye disease (TED), also known as thyroid-associated ophthalmopathy, Graves' ophthalmopathy, or Graves' orbitopathy, is a debilitating and painful autoimmune disease that can lead to serious facial appearance and visual-related changes and at times lead to blindness. Although the cause of thyroid eye disease is not fully understood [1], insulinlike growth factor 1 receptor (IGF-1R) overexpression and activation within orbital fibroblasts and $\mathrm{B}$ and $\mathrm{T}$ cells are thought to play a central pathogenic role [2-4]. A complex formed by the IGF-IR and thyrotropin receptor drives intracellular signaling, leading to hyaluronan accumulation, adipogenesis, and immune infiltration, resulting in inflammation, edema, and expansion of extraocular muscle and adipose tissue [5-8].

Teprotumumab, a fully human monoclonal antibody, binds specifically to IGF-1R with resultant cellular internalization and blockade of signaling mediated by IGF-1R, thereby blocking pathologic immune responses in TED. Teprotumumab has previously demonstrated statistically and clinically meaningful improvements in multiple outcomes of the disease in two randomized, placebo-controlled, double-masked, phase II and III trials $[9,10]$ and is now the first FDA-approved therapy for TED [11] (marketed as TEPEZZA [teprotumumab-trbw] for injection). The approved regimen for TED is an initial intravenous (IV) infusion of $10 \mathrm{mg} / \mathrm{kg}$ followed by $20 \mathrm{mg} / \mathrm{kg}$ every 3 weeks for an additional seven infusions. This regimen was selected to maintain pharmacologically active exposures and a greater than $90 \%$ saturation of IGF-1R during the dosing interval to achieve efficacy balanced with a well-tolerated safety profile for this vision-threatening disease.

As an IGF-1R inhibitor, teprotumumab (formerly known as RG1507) was initially evaluated in multiple oncology indications at doses from 1 to $27 \mathrm{mg} / \mathrm{kg}$ (e.g., clinicaltrials.gov number: NCT00400361, NCT00642941) prior to its development in TED. The maximum tolerated dose of teprotumumab was not achieved at the highest doses evaluated $(27 \mathrm{mg} / \mathrm{kg}$ every 3 weeks [q3w] (data on file) and $9 \mathrm{mg} /$ $\mathrm{kg}$ weekly [qw] [12]). The pharmacokinetic (PK), pharmacodynamic, and safety data of teprotumumab in oncology patients informed the development of teprotumumab for TED. Specifically, teprotumumab showed dose-dependent clearance (CL) decreasing from a mean of $812 \mathrm{~mL} /$ day at 1 $\mathrm{mg} / \mathrm{kg}$ to $418 \mathrm{~mL} / \mathrm{day}$ at $16 \mathrm{mg} / \mathrm{kg}$ [13], suggesting a saturable elimination pathway. An initial population PK model was built with these data to characterize the nonlinear pharmacokinetics of teprotumumab at this dose range and identify a concentration that saturates greater than $90 \%$ of targetmediated drug disposition, implying a greater than $90 \%$ saturation of IGF-1R [14]. This concentration was also set as the target trough concentration when selecting an expected safe therapeutic dose regimen of teprotumumab in TED. Additionally, it was found that total IGF-1 levels increased in a dose-dependent manner upon teprotumumab treatment [12], confirming target engagement at the doses of teprotumumab evaluated in oncology patients.

The PK/pharmacodynamic characteristics of teprotumumab in patients with TED have not been previously reported. Here, we report the PK characteristics and immunogenicity of teprotumumab, how the teprotumumab dose regimen was selected for clinical evaluation, and the supporting evidence that examined the relationship between teprotumumab exposures, efficacy, and safety in patients with TED.

\section{Methods}

\subsection{Studies}

Pharmacokinetic data from three studies were used to inform the approved teprotumumab dosing regimen for patients with TED. The first study was an open-label, multi-center, sequential-group, phase I dose-escalation study of teprotumumab in adults with advanced solid tumors, non-Hodgkin lymphoma, or Hodgkin lymphoma (clinicaltrials.gov number: NCT00400361) [12, 13]. Two IV dosing schedules along with dose escalations $(1,3,9$, and $16 \mathrm{mg} / \mathrm{kg} \mathrm{q} 3 \mathrm{w} ; 1,3$, and $9 \mathrm{mg} / \mathrm{kg} \mathrm{qw}$ ) were evaluated using drug material derived from the SP2/0 cell line. Chinese hamster ovary (CHO) cellderived teprotumumab, the same cell line used to produce the approved teprotumumab, was also evaluated at 3,6, and $9 \mathrm{mg} / \mathrm{kg} \mathrm{qw}$ and at $16 \mathrm{mg} / \mathrm{kg} \mathrm{q} 3 \mathrm{w}$. Pharmacokinetic samples were collected prior to dosing and at 15, 60, and $90 \mathrm{~min}$ and $6,24,48$, and $72 \mathrm{~h}$ following completion of the first infusion and the week 7 infusion (regardless of dosing schedule $\mathrm{q} 3 \mathrm{w}$ or qw). Additionally, PK samples were collected at cycle 1 days 8 and 15 , prior to dose on cycle 2 , cycle 3 day 8 , and cycle 4 day 1 for the $\mathrm{q} 3 \mathrm{w}$ cohorts; and prior to dosing on weeks $2,3,4,8$, and 10 for the qw cohorts.

The second study was a double-masked, randomized (1:1), placebo-controlled phase II study of teprotumumab in adult patients with active, moderate-to-severe thyroid eye disease (clinicaltrials.gov number: NCT01868997) [9]. Forty-three patients were randomized to teprotumumab and scheduled to receive IV infusions over 60-90 $\mathrm{min}$ at a dose of $10 \mathrm{mg} / \mathrm{kg}$ on day 1 , followed by seven doses at $20 \mathrm{mg} / \mathrm{kg}$ q3w starting from week 3. During the doublemasked treatment period, teprotumumab was administered on day 1 , weeks $3,6,9,12,15,18$, and 21 . A final visit was performed at week 24 . Following completion of the double-masked treatment period, subjects entered a 48 -week follow-up period with no additional treatment during the 
first 3 months, unless determined to be medically necessary. Serum PK samples were collected pre- and post-infusion at weeks 0,3 , and 9 , as well as at weeks 1,4 , and 24. Serum samples for anti-drug antibody (ADA) evaluation were collected prior to dosing at weeks $0,3,9,24,36$, and 72 .

The third study was a randomized (1:1 ratio; stratified by tobacco use status), double-masked, placebo-controlled, parallel-group phase III study in adult subjects with active, moderate-to-severe TED (clinical trials.gov number: NCT03461211) [10]. Forty-one patients with TED were randomized to teprotumumab and received eight infusions of teprotumumab $(10 \mathrm{mg} / \mathrm{kg}$ for the first infusion and $20 \mathrm{mg} /$ $\mathrm{kg}$ for the remaining seven infusions). The study comprised a 24-week double-masked treatment period (teprotumumab dosing on day 1 , week $3,6,9,12,15,18$ and 21), followed by a 48 -week follow-up period without further teprotumumab treatment for subjects who were proptosis responders and subjects who were non-responders but chose not to enroll in an open-label extension study. Only PK and ADA data from the double-masked treatment period were included in the analysis reported here. Pharmacokinetic samples were collected prior to and at the end of the infusions on day 1 , week 3 and week 9 of the treatment period; as well as at weeks 1,4 , and 24 . Anti-drug antibody samples were collected prior to dosing on weeks $0,3,9$, and 24 .

The trials were approved by institutional review boards of participating institutions and performed in accordance with the Declaration of Helsinki. Written informed consent was obtained from participants.

\subsection{Bioanalytical Assay Methods}

Validated bioanalytical assays were used to measure the serum concentrations of teprotumumab. An enzyme-linked immunosorbent assay method using streptavidin-coated plates incubated with biotinylated recombinant human IGF receptor was used to assay the serum PK samples from the phase I oncology study. The assay working range was $25-1000 \mathrm{ng} / \mathrm{mL}$. The inter- and intra-assay precision range was $6.2-7.8 \%$ and $1.6-14.2 \%$, respectively. The inter- and intra-assay accuracy range was $-6.8 \%$ to $1.3 \%$ and $-11 \%$ to $8.6 \%$, respectively.

An enzyme-linked immunosorbent assay method using plates coated with the human IGF-1R (Sino Biologial Inc. Cat.\# 10164-H08H) was utilized to assay PK samples from the phase II study. The calibration range was $76.3-1280 \mathrm{ng} /$ $\mathrm{mL}$. The inter- and intra-assay accuracy ranged from -6.1 to $1.9 \%$ and -16.9 to $16.7 \%$, respectively. The inter- and intra-assay precision ranges were $3.9-8.7 \%$ and $0-12.3 \%$, respectively.

An electrochemiluminescence assay was used to test phase III study serum PK samples. The range of quantification was from 10 to $4000 \mathrm{ng} / \mathrm{mL}$. The inter- and intra-assay precision ranges were $9.7-14.1 \%$ and $10.1-25.1 \%$, respectively. The inter- and intra-assay accuracy ranges were $-2.8 \%$ to $2.6 \%$ and $-4.6 \%$ to $11.2 \%$, respectively.

Validated electrochemiluminescence assay methods were used to detect the presence of ADAs in human serum in the phase II and III studies. For the Phase II study ADA assay, the drug tolerance level was $3.906 \mu \mathrm{g} / \mathrm{mL}$ at low quality control $(250 \mathrm{ng} / \mathrm{mL})$ and $250 \mu \mathrm{g} / \mathrm{mL}$ at high quality control $(4000 \mathrm{ng} / \mathrm{mL}$ ). The maximum drug tolerance at the tested antibody levels was $400 \mu \mathrm{g} / \mathrm{mL}$ for the phase III ADA assay.

\subsection{Population PK analysis}

To select the teprotumumab dose for phase II evaluation in TED, an initial population PK analysis was performed on PK data from the phase I oncology study using the SP2/0 material. In total, $816 \mathrm{PK}$ data points from 60 subjects receiving IV teprotumumab from 1 to $16 \mathrm{mg} / \mathrm{kg}$ were included in the analysis. A similar population PK analysis was performed utilizing all PK data from the phase I study to compare teprotumumab pharmacokinetics using the SP2/0 material and $\mathrm{CHO}$ material. The covariate effect was not evaluated in the analysis. Model parameter estimation and evaluation were implemented with NONMEM (version 6; ICON, College Park, MD, USA).

A final population PK analysis to support teprotumumab registration was performed utilizing pooled PK data using CHO-derived teprotumumab from the phase I oncology study and the phase II and III studies in TED. The robust PK data collected from doses 3 to $16 \mathrm{mg} / \mathrm{kg}$ in the phase I oncology study (CHO-derived material only) were included to inform the model structure and adequately characterize the pharmacokinetics of teprotumumab as only sparse PK samples were collected in the phase II and phase III TED studies. In total, 462 concentration data points from 36 subjects in the phase I oncology study and 598 samples from 83 subjects from the phase II and III studies in active TED were analyzed using nonlinear mixed-effect modeling. Observations below the lower limit of quantitation were omitted, as only $8.8 \%$ of data points were below the lower limit of quantitation and the censored-data likelihood method (M3 method in NONMEM) was not required [15].

Clinically relevant covariates were evaluated for their potential impact on teprotumumab pharmacokinetics, including baseline demographic covariates (age, weight [actual body weight], sex, race, ethnicity, and smoking status) and hepatic and renal function-related covariates (bilirubin, alanine aminotransferase, aspartate aminotransferase, and creatinine CL) [16] (Table 1).

Log-transformed teprotumumab concentration data were used in the population PK analysis. The best structural population PK model (final base model) was selected first after comparison of various structural models. A diagonal 
Table 1 Baseline population characteristics in the final population pharmacokinetic dataset

\begin{tabular}{lclll}
\hline Continuous covariates (unit) & Median [min, max $]$ & $N$ & Categorical covariates (group) & $N$ \\
\hline Age (years) & $52[18,80]$ & 119 & Race (white/Asian/black/other) & $103 / 3 / 10 / 3$ \\
Weight $(\mathrm{kg})$ & $74.8[45.8,169]$ & 119 & Sex (male/female) & $47 / 72$ \\
Bilirubin $(\mu \mathrm{mol} / \mathrm{L})$ & $8[2.74,24.3]$ & 116 & Smoking status (missing/non-user/user) & $36 / 63 / 20$ \\
AST (U/L) & $21[11,221]$ & 118 & Ethnicity (missing/non-Hispanic/Hispanic) & $2 / 113 / 4$ \\
ALT (U/L) & $20[7,174]$ & 119 & Disease (oncology/TED) & $36 / 83$ \\
Creatinine (mmol/L) & $0.814[0.441,1.8]$ & 119 & & \\
Creatinine clearance (mL/min) & $104[41.6,278]$ & 119 & & \\
\hline
\end{tabular}

$A L T$ alanine aminotransferase, $A S T$ aspartate aminotransferase, max maximum, $\min$ minimum, $T E D$ thyroid eye disease

$\Omega$ matrix was implemented to estimate random effects in the base PK model. Covariates were then tested for their impact on the model parameters from the base model. A step-wise forward addition and backward deletion model selection strategy was used, and linear as well as non-linear relationships between the explanatory covariates and model parameters were evaluated. Model selection was done based on a log likelihood ratio test at an acceptance $p$ value of 0.01 (forward addition) or 0.001 (backward elimination).

Robustness of the final population PK model was assessed using the diagnostic goodness-of-fit plots, prediction-corrected visual predictive check [17], numerical predictive check [18], and non-parametric bootstrap [19, 20]. Shrinkage was also assessed for the between-subject random effects $(\eta)$ and the residual random effect $(\varepsilon)$ of the final model.

Model parameter estimation and evaluation were implemented with NONMEM 7 (version 7.3.0, ICON) with Intel (R) Fortran Compiler (version 10.1.021), Perl Speaks NONMEM (version 3.2.12, Uppsala University, Uppsala, Sweden), and R 3.3.1. Population PK estimation was performed using the first-order conditional estimation with interaction method in NONMEM.

The model-predicted exposure metrics at steady state (steady-state area under the concentration-time curve [AUC ss $]$, peak $\left[C_{\text {max,ss }}\right]$, and trough $\left[C_{\text {min,ss }}\right]$ concentrations) were computed using the Bayesian post-hoc PK parameters following administration of teprotumumab and merged into the efficacy and safety datasets for exposure-response analysis. Additionally, a lower dose (10 $\mathrm{mg} / \mathrm{kg} \mathrm{q} 3 \mathrm{w})$ and less frequent dosing ( $20 \mathrm{mg} / \mathrm{kg}$ every 4 weeks [q4w]) were simulated in comparison to the approved teprotumumab regimen. These analyses were implemented with R3.5.1.

\subsection{Exposure-Response Analysis}

The exposure-response relationships for both efficacy and safety endpoints were explored based on the data from the phase II and phase III studies in patients with TED. The total number of teprotumumab-treated patients included in the analysis was 83 .
Key efficacy endpoints included proptosis response (defined as a reduction in proptosis of $\geq 2 \mathrm{~mm}$ in the study eye) at week 24; clinical activity score (CAS) categorical response (defined as a CAS of 0 or 1 , indicating no or minimal inflammation, respectively) at week 24; and diplopia response (defined as a reduction in diplopia of $\geq 1$ grade from baseline) in subjects who had diplopia at baseline. For the week 24 diplopia responder rate, 66 teprotumumabtreated subjects with baseline diplopia were included. Key safety variables assessed included hyperglycemia, muscle spasm, and hearing impairment. All efficacy endpoints and safety variables were binary responses (yes/no).

The model-predicted steady-state exposures of teprotumumab $\left(\mathrm{AUC}_{\mathrm{ss}}, C_{\mathrm{max}, \mathrm{ss}}\right.$, and $\left.C_{\mathrm{min}, \mathrm{ss}}\right)$ were used for the exposure-response analysis. Specifically, scatterplots of teprotumumab exposures stratified by endpoint values were generated, the probability of response was calculated across sets of patients binned by teprotumumab exposure quantiles, and last, logistic regression models were developed for all efficacy endpoints, and safety variables. An exposureresponse analysis was performed with R 3.5.1.

\section{Results}

\subsection{Dose Selection for Thyroid Eye Disease}

The initial population PK analysis was performed on teprotumumab PK data from 60 oncology patients, aged 18-81 years, of whom 21 were women, with a weight range from 48.5 to $126.1 \mathrm{~kg}$. A two-compartment model with a parallel elimination process (linear and nonlinear CL) from the central compartment best described the PK data. The linear (or nonspecific) CL (estimated to be $0.34 \mathrm{~L} /$ day) is mediated by interaction between the $\mathrm{Fc}$ region of the monoclonal antibody and $\mathrm{Fc}$ receptors, and the nonlinear (or specific) $\mathrm{CL}$ is mediated by the specific interaction between the Fab region of the monoclonal antibody and its antigenic targets [21] (i.e., target-mediated disposition). The nonlinear CL was described by a Michaelis-Menten process characterized 
by a maximum metabolic rate $\left(V_{\max }\right)$ of $3.02 \mathrm{mg} /$ day and a Michaelis-Menten constant of $1.5 \mu \mathrm{g} / \mathrm{mL}$. Based on this model, $20 \mu \mathrm{g} / \mathrm{mL}$ was selected as the target trough concentration required to maintain a greater than $90 \%$ saturation of target-mediated drug disposition, which implies a greater than $90 \%$ saturation of IGF-1R. The population parameter estimates were $3.3 \mathrm{~L}$ for the volume of central compartment $\left(V_{\mathrm{c}}\right), 0.70 \mathrm{~L} / \mathrm{h}$ for the intercompartment CL $(\mathrm{Q})$, and $3.5 \mathrm{~L}$ for the volume of peripheral compartment $\left(V_{\mathrm{p}}\right)$. The between-subject variability was estimated as $38 \%$ on CL, $20 \%$ on $V_{\mathrm{c}}$, and $59 \%$ on $V_{\mathrm{p}}$. The residual (unexplained) variability was $17 \%$.

A further population analysis with the same PK model using pooled PK data from SP2/0 and CHO material in the phase I oncology study indicated that CHO-derived teprotumumab exhibited a modest decrease in exposure $(\sim 30 \%)$ compared with the SP2/0-derived teprotumumab. As such, the teprotumumab dose regimen (an initial dose of $10 \mathrm{mg} / \mathrm{kg}$ followed by $20 \mathrm{mg} / \mathrm{kg} \mathrm{q} 3 \mathrm{w}$ ) was chosen to maintain the target trough concentration of $20 \mathrm{ug} / \mathrm{mL}$ over the dosing interval in patients with TED using the CHO-derived material.

\subsection{Demographics and Baseline Characteristics for Final Population PK Analysis}

Baseline demographic and laboratory parameters that were evaluated as potential covariates on teprotumumab pharmcokinetics from 36 oncology patients and 83 patients with active TED from the phase II and III studies are summarized in Table 1 . The median age was 52 years (range: $18-80$ years) and the median weight was $74.8 \mathrm{~kg}$ (range 45.8-169 $\mathrm{kg}$ ). There were more female subjects ( 72 female vs 47 male) and majority of the population was white.

\subsection{Final Population PK Results}

Serum pharmacokinetics of teprotumumab (CHO derived) in oncology and patients with TED (dose range 3-20 mg/kg) can be adequately described by a two-compartment model with first-order elimination from the central compartment and redistribution from the peripheral compartment. A summary of key population PK parameters is presented in Table 2. The model-estimated typical PK parameters were $0.334 \mathrm{~L} /$ day for $\mathrm{CL}, 3.94 \mathrm{~L}$ for $V_{\mathrm{c}}, 0.859 \mathrm{~L} /$ day for $Q$, and $4.21 \mathrm{~L}$ for $V_{\mathrm{p}}$. Inter-individual variability on $\mathrm{CL}, V_{\mathrm{c}}, Q$, and $V_{\mathrm{p}}$ were $43.0 \%, 27.7 \%, 47.8 \%$, and $25.4 \%$, respectively. The geometric mean elimination half-life was 19.9 days $(26.2 \%$ coefficient of variation) in patients with TED.

Sex was identified as a statistically significant covariate on teprotumumab pharmacokinetics: with female subjects having lower $V_{\mathrm{c}}$ than male subjects $(3.25 \mathrm{~L}$ and 3.94 $\mathrm{L}$, respectively). The sensitivity analysis in patients with TED after repeated doses of teprotumumab showed that the covariate effect of sex on teprotumumab $V_{\mathrm{c}}$ resulted in similar $\mathrm{AUC}_{\mathrm{ss}}, 14.5 \%$ higher $C_{\mathrm{max}, \mathrm{ss}}$, and $4.91 \%$ lower $C_{\text {min,ss }}$ in female compared with male patients, and these modest differences are not considered to be clinically meaningful. Other covariates, including baseline age, body weight, ethnicity, race, bilirubin, aspartate aminotransferase, alanine aminotransferase, creatinine $\mathrm{CL}$, and smoking status did not show a statistically significant impact on the pharmacokinetics of teprotumumab.

Model diagnostic assessments confirm that the final population PK model adequately described the teprotumumab PK data and was robust. Goodness-of-fit plots showed a good agreement between the predicted concentrations and the observed concentrations; and no apparent bias in the

Table 2 Population PK parameters of teprotumumab and bootstrap results

\begin{tabular}{|c|c|c|}
\hline Parameter description & $\begin{array}{l}\text { Final population PK model } \\
\text { Median (2.5th-97.5th percentile) }\end{array}$ & $\begin{array}{l}\text { Bootstrap estimates }{ }^{\mathrm{a}} \\
\text { Median }\left(2.5 \text { th }-97.5^{\text {th }} \text { percentile }\right)\end{array}$ \\
\hline Central clearance, $\mathrm{CL}$ (L/day) & $0.334(0.324-0.345)$ & $0.334(0.304-0.362)$ \\
\hline Central volume, $V_{\mathrm{c}}(\mathrm{L})$ & $3.94(3.65-4.26)$ & $3.94(3.67-4.26)$ \\
\hline Inter-compartmental clearance, $\mathrm{Q}$ (L/day) & $0.859(0.85-0.869)$ & $0.852(0.641-1.17)$ \\
\hline Peripheral volume, $V_{\mathrm{p}}(\mathrm{L})$ & $4.21(4.14-4.27)$ & $4.23(3.89-4.69)$ \\
\hline Influence of sex on $V_{c}$ & $-0.191(-0.246$ to -0.136$)$ & $-0.190(-0.284$ to -0.0968$)$ \\
\hline IIV on CL (\%) & $43.0(36.0-49.0)$ & $42.7(36.5-48.7)$ \\
\hline IIV on $V_{\mathrm{c}}(\%)$ & $27.7(23.3-31.5)$ & $27.6(23.4-31.4)$ \\
\hline IIV on Q $(\%)$ & $47.8(37.5-56.3)$ & $47.3(27.8-61.0)$ \\
\hline $\operatorname{IIV} V_{\mathrm{p}}(\%)$ & $25.4(12.1-33.8)$ & $24.6(10.5-33.6)$ \\
\hline Covariance between CL and $V_{\mathrm{c}}$ & $0.0763(0.0472-0.105)$ & $0.0759(0.0467-0.106)$ \\
\hline Residual error $(\%)$ & $18.0(15.4-20.2)$ & $18.0(15.7-20.4)$ \\
\hline
\end{tabular}

$I I V$ inter-individual variability, $P K$ pharmacokinetic

${ }^{\mathrm{a}}$ Based on results from 1000 bootstrap runs 
residuals plots over time and across predicted concentration, with only a very small portion of data points showing conditional weighted residuals (ICWRESI) greater than 2 (Fig. 1 of the Electronic Supplementary Material [ESM]).

Bootstrap results confirm that the final population PK model was stable with good precision of parameter estimation. As shown in Table 2, median values of bootstrapping were very similar to the parameter estimates of the final model and the 2.5th-97.5th percentile ranges of the final model estimates and of the bootstrapping results overlapped.

Both prediction-corrected visual predictive check plots (Fig. 2 of the ESM) and numerical predictive check results suggest that the model adequately describes the central tendency and variability of teprotumumab concentrations. Specifically, $47.7 \%, 22 \%$, and $4.06 \%$ of the observations were above the 50th, 75th, and 95th percentile of the model predictions, respectively. Furthermore, $52.3 \%, 25.8 \%$, and $6.79 \%$ of the observations were below the 50th, 25 th, and 5th percentile of the model predictions, respectively.

The magnitude of shrinkage was considered small enough for CL and $V_{\mathrm{c}}(<10 \%)$, thus the final population PK model generated reliable Bayesian estimations for CL and $V_{\mathrm{c}}$ and was robust to describe the relationship between $\mathrm{CL} / V_{\mathrm{c}}$ and the related covariates. Volume of peripheral compartment and $Q$ had greater $\eta$-shrinkage of higher than $40 \%$ in the final model, suggesting that $\mathrm{PK}$ data might be insufficient to adequately characterize the inter-subject variability of those parameters in the final model.

\subsection{Simulated Teprotumumab Exposures in Patients with TED}

After an initial dose of $10 \mathrm{mg} / \mathrm{kg}$ followed by seven repeated doses of $20 \mathrm{mg} / \mathrm{kg} \mathrm{q} 3 \mathrm{w}$, population PK model-predicted exposures of teprotumumab in patients with TED are as follows: mean ( \pm standard deviation) values for $\mathrm{AUC}_{\mathrm{ss}}, C_{\text {max,ss }}$, and $C_{\text {min,ss }}$ are $131( \pm 30.9) \mathrm{mg} \cdot \mathrm{h} / \mathrm{mL}, 643( \pm 130) \mu \mathrm{g} / \mathrm{mL}$, and $157( \pm 50.6) \mu \mathrm{g} / \mathrm{mL}$, respectively. The predicted minimum trough concentration of teprotumumab is $21.9 \mu \mathrm{g} / \mathrm{mL}$ after the first infusion and $65.7 \mu \mathrm{g} / \mathrm{mL}$ at steady state, confirming that trough concentrations were consistently above $20 \mu \mathrm{g} / \mathrm{mL}$, the concentration predicted to be associated with greater than $90 \%$ IGF-1R saturation, in all patients.

\subsection{Exposure-Response Analysis Results}

Overall, there were no meaningful correlations between teprotumumab exposures and key clinical efficacy endpoints (proptosis, diplopia, and CAS responses) or safety variables (hyperglycemia, muscle spasms, and hearing impairment), supporting the selected teprotumumab regimen (an initial infusion of $10 \mathrm{mg} / \mathrm{kg}$ followed by $20 \mathrm{mg} / \mathrm{kg} \mathrm{q} 3 \mathrm{w}$ for a total of eight infusions) as appropriate for the treatment of TED.
Because of the lack of a meaningful exposure-response relationship between teprotumumab exposures and the efficacy and safety endpoints evaluated, no further covariate (e.g., weight, age, sex, or smoking status) analysis on exposureresponse relationships were performed.

\subsection{Proptosis Response}

The scatter plots in Fig. 1a depict the distribution of exposures in teprotumumab-treated subjects with or without proptosis responses at week 24 . The median $\mathrm{AUC}_{\mathrm{ss}}$ and $C_{\text {max,ss }}$ of teprotumumab were comparable between responders and non-responders, and the median $C_{\text {min,ss }}$ was slightly lower for non-responders. There were considerable overlaps in the ranges of $\mathrm{AUC}_{\mathrm{ss}}, C_{\mathrm{max}, \mathrm{ss}}$, and $C_{\mathrm{min}, \mathrm{ss}}$ between the two response groups.

The lowest quartile of $C_{\text {min,ss }}$ from the approved teprotumumab regimen, while highly efficacious, was associated with a slightly lower numeric proptosis response (66.7\%), about 13-19\% lower compared with the higher three quartiles (80-85.7\%) (Table 3). Further logistic regression models for the proptosis response rate with $\mathrm{AUC}_{\mathrm{ss}}, C_{\text {max,ss }}$, or $C_{\text {min,ss }}$ suggested no significant correlation between proptosis response and $\mathrm{AUC}_{\mathrm{ss}}$ or $C_{\mathrm{max}, \mathrm{ss}}$ (data not shown), but a statistically significant association between proptosis responder rate and $\mathrm{C}_{\text {min,ss }}$ ( $p$-value of 0.037 for slope, Fig. 2). However, the difference in the observed proptosis responder rate over the $C_{\text {min,ss }}$ quartiles was less than $20 \%$, and the observed proptosis responder rate for the lowest $C_{\text {min,ss }}$ quartile group (66.7\%) was still substantially higher than the placebo group (14.9\%).

With a lower dose or a less frequent dose, the majority of patients will have exposures at or below the lowest quartile $C_{\text {min,ss }}$, which may result in a lower proptosis response. Specifically, $10 \mathrm{mg} / \mathrm{kg} \mathrm{q} 3 \mathrm{w}$ and $20 \mathrm{mg} / \mathrm{kg} \mathrm{q} 4 \mathrm{w}$ will result in $91 \%$ and $72 \%$ subjects, respectively, with $C_{\min , \mathrm{ss}}$ lower than the first quartile $\mathrm{C}_{\text {min,ss }}$ of the teprotumumab regimen for TED.

\subsection{CAS and Diplopia Response}

The scatter plots in Fig. 1b depict the distribution of exposures in teprotumumab-treated subjects who were CAS categorical responders or non-responders at week 24 . Similarly, Fig. 1c depicts the distribution of exposures in teprotumumab-treated subjects with or without diplopia responses at week 24. For both CAS and diplopia responses, the median values of $\mathrm{AUC}_{\mathrm{ss}}, C_{\mathrm{max}, \mathrm{ss}}$, and $C_{\mathrm{min}, \mathrm{ss}}$ were comparable between responders and non-responders, and the interquartile range and overall range of $\mathrm{AUC}_{\mathrm{ss}}, C_{\mathrm{max}, \mathrm{ss}}$, and $C_{\text {min,ss }}$ were also similar between the two response groups. The probability of the CAS or diplopia responses by quantiles of teprotumumab exposures $\left(\mathrm{AUC}_{\mathrm{ss}}, C_{\mathrm{max}, \mathrm{ss}}\right.$, and $\left.C_{\text {min,ss }}\right)$ showed no clear trend of correlation (Table 3). Logistic regression of CAS or diplopia response vs $\mathrm{AUC}_{\mathrm{ss}}, C_{\mathrm{max}, \mathrm{ss}}$, 

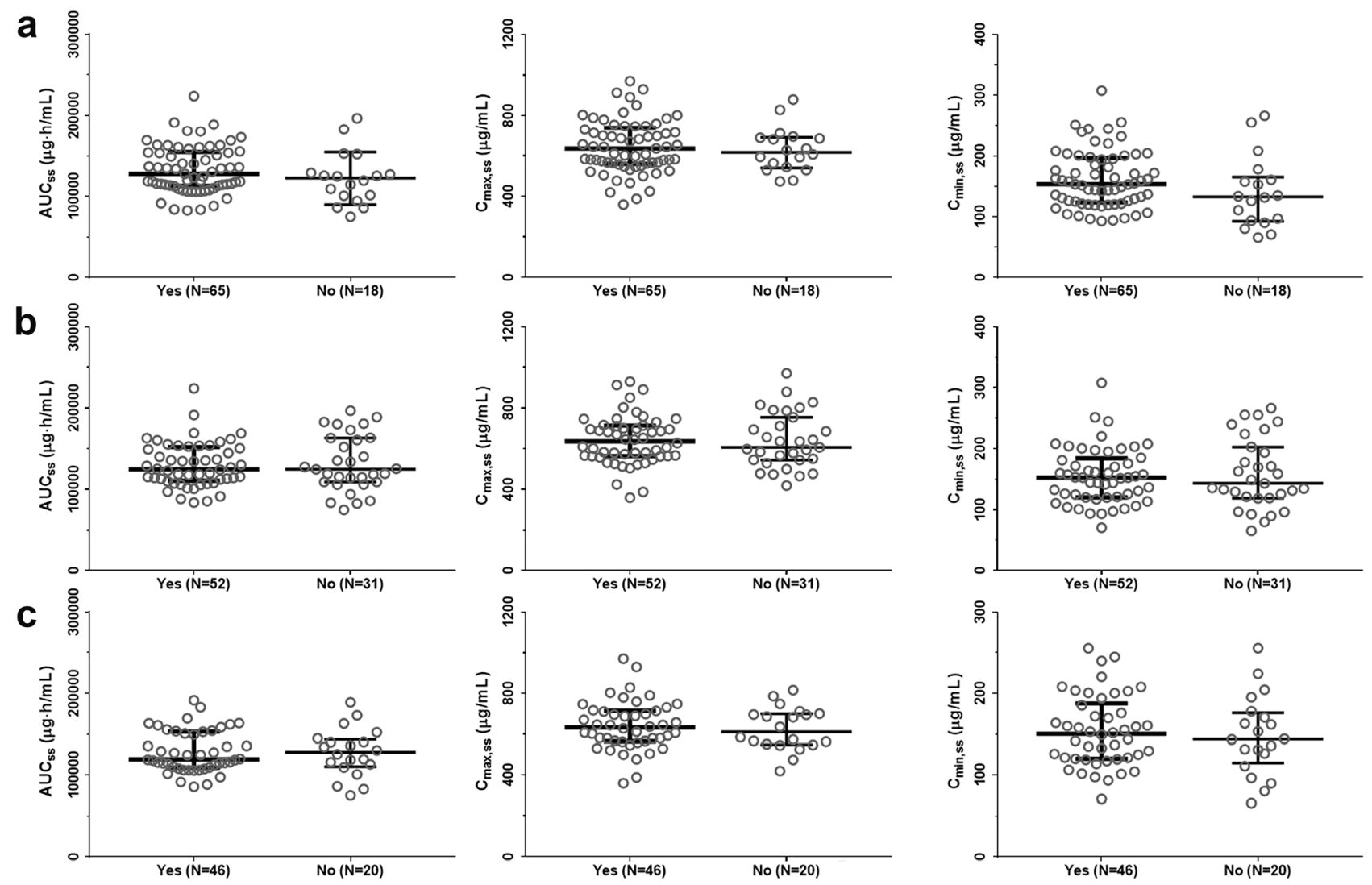

Fig. 1 Exposure-response relationship for key efficacy endpoints at week 24. Panel a, proptosis response; panel b, clinical activity score response; panel c, diplopia response. Open circles represent each subject's exposure; lines represent median and interquartile ranges.

and $C_{\text {min,ss }}$ did not show a significant association between CAS or diplopia response rate and teprotumumab exposures (data not shown).

\subsection{Exposure-Safety Analysis}

The scatter plots (Fig. 3) depict the distribution of steadystate exposures in teprotumumab-treated subjects with or without hyperglycemia, muscle spasms, or hearing impairment. The median values, the interquartile range, and the overall range of teprotumumab exposures $\left(\mathrm{AUC}_{\mathrm{ss}}, C_{\text {max,ss }}\right.$, and $C_{\text {min,ss }}$ ) were similar between subjects with and without hyperglycemia, muscle spasms, or hearing impairment following teprotumumab treatment. The probability of muscle spasms or hearing impairment by quantiles of teprotumumab exposures showed no exposure-response relationship (Table 3). Further logistic regression of muscle spasms or hearing impairment vs $\mathrm{AUC}_{\mathrm{ss}}, C_{\mathrm{max}, \mathrm{ss}}$, and $C_{\text {min,ss }}$ confirmed the lack of significant association between teprotumumab
$\mathrm{AUC}_{\mathrm{ss}}$ steady-state area under the concentration-time curve, $C_{\text {max.ss }}$ steady-state peak concentration, $C_{\mathrm{min}, \mathrm{ss}}$ steady-state trough concentration

exposures and the incidence of muscle spasms or hearing impairment (data not shown). The probability of hyperglycemia showed a slightly higher incidence in the third and fourth quantiles of $\mathrm{AUC}_{\mathrm{ss}}$, but no clear difference across quantiles of $C_{\max , s s}$ and $C_{\text {min,ss. }}$. Nonetheless, further logistic regression showed no significant association between teprotumumab exposures $\left(\mathrm{AUC}_{\mathrm{ss}}, C_{\mathrm{max}, \mathrm{ss}}\right.$, and $\left.C_{\mathrm{min}, \mathrm{ss}}\right)$ and the incidence of hyperglycemia.

\subsection{Immunogenicity in Subjects with TED}

Overall, teprotumumab showed low immunogenicity. Among the 83 patients with TED who received teprotumumab treatment, only two subjects were confirmed ADA positive with no detectable titer (potential false positive): one at week 3 and another at baseline and week 72. Both subjects had PK profiles comparable to those of ADAnegative subjects, confirming a lack of clinically relevant immunogenicity with teprotumumab treatment. 
Table 3 Summary of efficacy and safety endpoints by exposure quantiles

\begin{tabular}{|c|c|c|c|c|c|}
\hline \multirow[t]{2}{*}{ Endpoints } & \multirow[t]{2}{*}{ Exposure } & \multicolumn{4}{|c|}{$\%$ of responder (yes/no) by exposure quantiles } \\
\hline & & $\mathrm{Q} 1(n=21)$ & $\mathrm{Q} 2(n=20)$ & $\mathrm{Q} 3(n=21)$ & $\mathrm{Q} 4(n=21)$ \\
\hline \multirow[t]{3}{*}{ Proptosis response } & $\mathrm{AUC}_{\mathrm{ss}}$ & $66.7(14 / 7)$ & $90(18 / 2)$ & $66.7(14 / 7)$ & $90.5(19 / 2)$ \\
\hline & $C_{\max , \mathrm{ss}}$ & $76.2(16 / 5)$ & $80(16 / 4)$ & $66.7(14 / 7)$ & $90.5(19 / 2)$ \\
\hline & $C_{\min , \mathrm{ss}}$ & $66.7(14 / 7)$ & $80(16 / 4)$ & $81.0(17 / 4)$ & $85.7(18 / 3)$ \\
\hline \multirow[t]{3}{*}{ Diplopia response } & $\mathrm{AUC}_{\mathrm{ss}}$ & $57.1(12 / 5)$ & $65(13 / 4)$ & $47.6(10 / 8)$ & $52.4(11 / 3)$ \\
\hline & $C_{\max , \mathrm{ss}}$ & $47.6(10 / 6)$ & $60(12 / 4)$ & $57.1(12 / 7)$ & $57.1(12 / 3)$ \\
\hline & $C_{\min , \mathrm{ss}}$ & $57.1(12 / 5)$ & $55(11 / 6)$ & $57.1(12 / 5)$ & $52.4(11 / 4)$ \\
\hline \multirow[t]{3}{*}{ CAS response } & $\mathrm{AUC}_{\mathrm{ss}}$ & $57.1(12 / 9)$ & $70(14 / 6)$ & $66.7(14 / 7)$ & $57.1(12 / 9)$ \\
\hline & $C_{\max , \mathrm{ss}}$ & $52.4(11 / 10)$ & $70(14 / 6)$ & $66.7(14 / 7)$ & $61.9(13 / 8)$ \\
\hline & $C_{\min , \mathrm{ss}}$ & $61.9(13 / 8)$ & $55(11 / 9)$ & $76.2(16 / 5)$ & $57.1(12 / 9)$ \\
\hline \multirow[t]{3}{*}{ Hyperglycemia } & $\mathrm{AUC}_{\mathrm{ss}}$ & $4.8(1 / 20)$ & $5(1 / 19)$ & $14.3(3 / 18)$ & $14.3(3 / 18)$ \\
\hline & $C_{\max , \mathrm{ss}}$ & $9.5(2 / 19)$ & $0(0 / 20)$ & $19.0(4 / 17)$ & $9.5(2 / 19)$ \\
\hline & $C_{\min , \mathrm{ss}}$ & $4.8(1 / 20)$ & $10(2 / 18)$ & $9.5(2 / 19)$ & $14.3(3 / 18)$ \\
\hline \multirow[t]{3}{*}{ Muscle spasms } & $\mathrm{AUC}_{\mathrm{ss}}$ & $23.8(5 / 16)$ & $35(7 / 13)$ & $19.0(4 / 17)$ & $23.8(5 / 16)$ \\
\hline & $C_{\max , \mathrm{ss}}$ & $28.6(6 / 15)$ & $30(6 / 14)$ & $23.8(5 / 16)$ & $19.0(4 / 17)$ \\
\hline & $C_{\min , \mathrm{ss}}$ & $14.3(3 / 18)$ & $35(7 / 13)$ & $23.8(5 / 16)$ & $28.6(6 / 15)$ \\
\hline \multirow[t]{3}{*}{ Hearing impairment } & $\mathrm{AUC}_{\mathrm{ss}}$ & $9.5(2 / 19)$ & $15(3 / 17)$ & $4.8(1 / 20)$ & $9.5(2 / 19)$ \\
\hline & $C_{\mathrm{max}, \mathrm{ss}}$ & $14.3(3 / 18)$ & $15(3 / 17)$ & $0(0 / 21)$ & $9.5(2 / 19)$ \\
\hline & $C_{\min , \mathrm{ss}}$ & $9.5(2 / 19)$ & $10(2 / 18)$ & $14.3(3 / 18)$ & $4.8(1 / 20)$ \\
\hline
\end{tabular}

$A U C_{s s}$ steady-state area under the concentration-time curve, $C A S$ clinical activity score, $C_{\text {max }, s s}$ steady-state peak concentration, $C_{\text {min,ss }}$ steady-state trough concentration

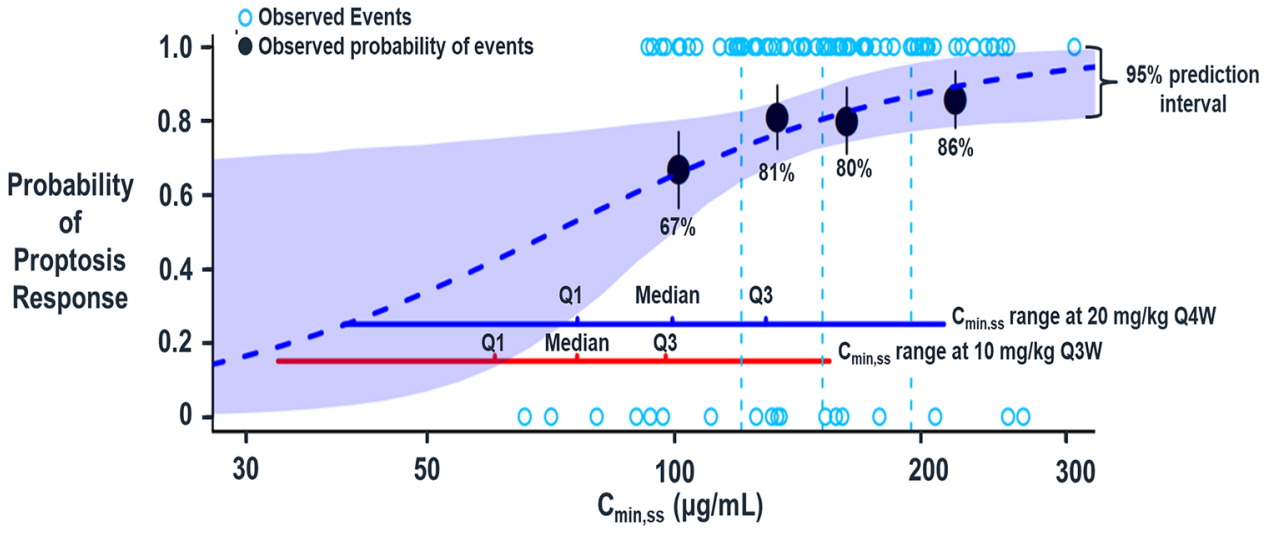

Fig. 2 Probability of proptosis response at week 24 vs steady-state trough concentration $\left(C_{\text {min,ss }}\right)$. Probability of proptosis response: $=1$ for responder; $=0$ for non-responder. The dark blue dashed line is the predicted probability by a linear logistic regression model. The shaded area is the $95 \%$ prediction interval based on 1000 bootstrap samples. The vertical light-blue dashed lines are the first quartile, median, and third quartile $C_{\text {min,ss }}$ (from left to right) following the

\section{Discussion}

Thyroid eye disease is a serious, debilitating, and painful autoimmune disease and teprotumumab is the first US Food and Drug Administration-approved therapy for this disease. The selected dose regimen was highly efficacious teprotumumab regimen for thyroid eye disease. The open blue circles reflect the observed events. The filled black symbols are the observed probability of events and the error bars are SE [sqrt $(P \times(1-P) / N)]$ for quantiles (at $100 \times(1 / q)$ th percentiles, vertical dotted lines) of exposures (plotted at the median value within each quantile). $Q 3 W$ every 3 weeks, $Q 4 W$ every 4 weeks

with regard to proptosis, clinical activity score, diplopia, and quality of life compared with placebo, and was generally well tolerated [10], supporting the approval of this regimen for the treatment of TED.

As a fully human monoclonal antibody, teprotumumab shows PK properties similar to that of other monoclonal 

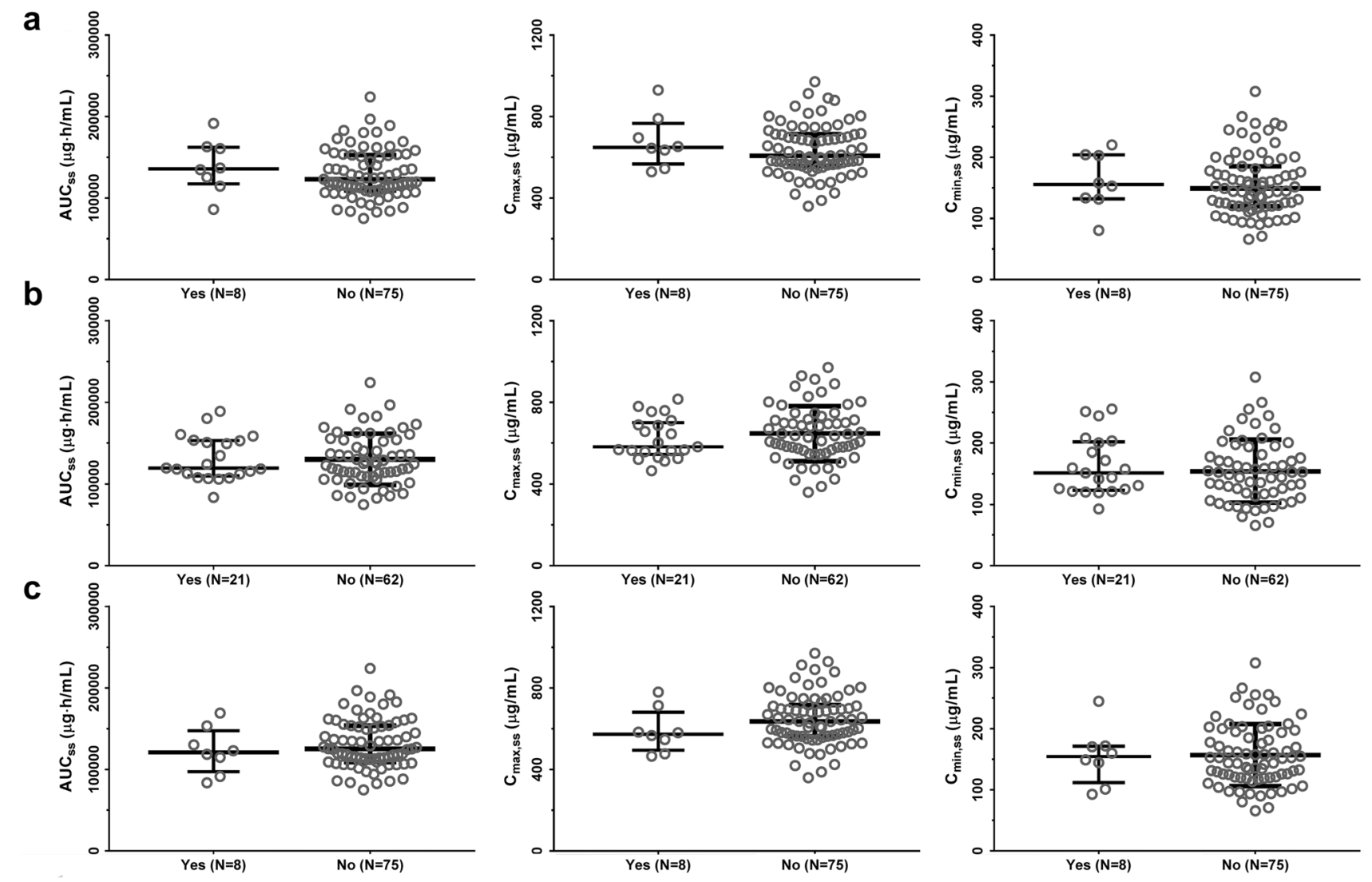

Fig. 3 Lack of correlation between teprotumumab exposure and selected safety variables. a hyperglycemia; b muscle spasms; c hearing impairment. Open circles represent each subject's exposure; lines

represent median and interquartile ranges. $A U C_{s s}$ steady-state area under the concentration-time curve, $C_{\max , s s}$ steady-state peak concentration, $C_{m i n, s s}$ steady-state trough concentration

antibodies and is characterized by a limited volume of distribution, slow CL, and a prolonged half-life [22, 23]. The initial population PK modeling using data from oncology studies that examined doses from 1 to $16 \mathrm{mg} / \mathrm{kg}$ in oncology patients suggested dual elimination pathways (target-mediated CL plus nonspecific CL) for teprotumumab. However, a two-compartment model with a linear CL pathway was adequate to describe the teprotumumab PK profile using data obtained with 3-20 mg/ $\mathrm{kg}$ doses from both oncology patients and patients with TED following single and multiple IV infusions. These findings suggest that target-mediated drug disposition was saturated at higher doses of teprotumumab ( $3 \mathrm{mg} / \mathrm{kg}$ and above) and the pharmacokinetics becomes linear at the clinical dose range $(10-20 \mathrm{mg} / \mathrm{kg})$ in patients with TED.

An important factor to consider for a monoclonal antibody directed against a specific target is the degree of target saturation during drug treatment [24]. The initial population PK modeling on data from 1 to $16 \mathrm{mg} / \mathrm{kg}$ of teprotumumab in the oncology study suggested the contribution of target-mediated CL, and a Michaelis-Menten constant of $1.5 \mu \mathrm{g} / \mathrm{mL}$. Thus, $20 \mu \mathrm{g} / \mathrm{mL}$ was selected as the target trough concentration required to maintain a greater than 90\% saturation of target-mediated drug disposition, which implies a greater than $90 \%$ saturation of IGF-1R. This value was also well above the pharmacologically active concentration of teprotumumab where $0.15-1 \mu \mathrm{g} / \mathrm{mL}$ blocked the interaction of IGF-1R and its ligand by $50 \%$ based on in vitro evaluations. For instance, $0.15 \mu \mathrm{g} / \mathrm{mL}$ of teprotumumab blocked IGF-1R autophosphorylation in mouse $3 \mathrm{~T} 3$ fibroblasts overexpressing human IGF-1R by $50 \%$; 1 $\mu \mathrm{g} / \mathrm{mL}$ of teprotumumab reduced cell surface IGF-1R levels in H322M lung cancer cells by $50 \%$ (data on file). A $20-\mathrm{mg} /$ $\mathrm{kg} \mathrm{q} 3 \mathrm{w}$ regimen following an initial dose of $10 \mathrm{mg} / \mathrm{kg}$ was thus selected for phase II and phase III evaluations as it was expected to maintain trough concentrations above $20 \mu \mathrm{g} /$ $\mathrm{mL}$ in the majority of patients with TED. The final population PK analysis confirmed the selected regimen achieved through concentrations consistently above $20 \mu \mathrm{g} / \mathrm{mL}$ in all patients. This regimen was highly effective and well tolerated in the treatment of TED and the lack of a meaningful exposure-response relationships for both efficacy and safety endpoints supports the demonstrated favorable benefit-risk profile of the TED dose regimen. It is noteworthy that $90 \%$ 
saturation of target-mediated drug disposition has been used for dose selection of other monoclonal antibodies as well, when direct or indirect target occupation measurement was not feasible or not available. For example, the dose selection of panitumumab, a monoclonal antibody against epidermal growth factor receptor, was also based on a similar approach [14].

Direct target engagement was not evaluated in patients with TED, but the pharmacodynamic effect of teprotumumab was evaluated by measuring total serum IGF-1 levels in oncology patients during early clinical development. Based on the mechanism of action for teprotumumab, the total IGF-1 level is expected to rise upon treatment with teprotumumab as the binding of IGF-1 to its receptor (IGF$1 \mathrm{R})$ is blocked. Total serum IGF-1 levels were increased (range 100-350\%) post teprotumumab treatment at all doses and regimens tested in oncology patients $(1-16 \mathrm{mg} / \mathrm{kg} \mathrm{q} 3 \mathrm{w}$ and $1-9 \mathrm{mg} / \mathrm{kg} \mathrm{qw}$ ) [12], suggesting that teprotumumab blocked the binding of IGF-1 to its receptor IGF-1R at the clinical doses evaluated, confirming target engagement following teprotumumab treatment.

It should be acknowledged that no dose-ranging studies of teprotumumab were conducted in TED, thus the lack of a meaningful exposure-response relationship for key efficacy and safety endpoints was concluded utilizing data from the approved teprotumumab regimen only, where teprotumumab was confirmed to be efficacious and well tolerated in patients with TED. Despite this limitation, the lowest quartile of $\mathrm{C}_{\text {min,ss }}$, although still highly effective, was associated with a numerically lower proptosis response, about 13-19\% lower compared with the higher three quantiles (80-85.7\%). Therefore, with a lower dose or a less frequent dose of teprotumumab, the majority of patients will have exposures at or below the lowest quartile $\mathrm{C}_{\text {min,ss }}$ from the current regimen, which may result in a lower proptosis response.

Based on the initial population PK analysis, weight-based dosing appeared to stabilize the exposure over body weight ranges and weight-based dosing was evaluated in both phase II and III studies. In the final population PK analysis, sex but not weight was identified as a significant covariate on the teprotumumab PK parameter $V_{\mathrm{c}}$ following the pre-specified covariate analysis criteria. The impact of weight and sex on pharmacokinetics could be confounded due to the correlation of weight and sex, however, the impact of sex on teprotumumab pharmacokinetics was minimal and therefore, no further evaluation was attempted.

Smoking is an important risk factor for TED, with the risk of TED increasing seven to eight times in smokers [25]. Thus, randomization was stratified by tobacco use status at baseline in both phase II and phase III TED studies. The treatment benefit of teprotumumab was observed in tobacco users and non-users and in both the study eye and fellow eye. Seventy percent of smokers were proptosis responders, with a mean reduction of $2.99 \mathrm{~mm}$ in the study eye [26]. These data are similar to results attained in non-smokers. Additionally, with regard to safety, tobacco use status did not suggest any clinically relevant differences from the overall analysis of treatment-emergent adverse events after teprotumumab treatment. This is consistent with the population PK analysis results where smoking was not a covariate impacting teprotumumab pharmacokinetics (i.e., similar teprotumumab exposures between tobacco users and non-users [detailed data not shown]), which is as expected because the CL of monoclonal antibodies is not subject to modulation of drugmetabolizing enzymes by smoking.

It is worth noting that teprotumumab exposures in patients with TED reported here are slightly different from the values reported in the approved US Food and Drug Administration label. For example, the mean ( \pm standard deviation) of AUC ss is $131( \pm 30.9) \mathrm{mg} \bullet \mathrm{h} / \mathrm{mL}$ here vs $138( \pm 34) \mathrm{mg} \bullet \mathrm{h} / \mathrm{mL}$ in the label. The reason is that only phase III PK data $(n=40)$ were included in the label. Some of the PK samples in the phase II study were assayed outside of the long-term stability range of the teprotumumab PK assay at the time the study was conducted, thus PK data from the phase II study were not included in the label. However, data from the phase II study are considered reliable and appropriate for inclusion in the overall PK analyses based on the known stability of antibodies when stored under appropriate frozen conditions [27]; consistent with this, the pharmacokinetics of teprotumumab were similar between phase II and phase III studies (Table 1 of the ESM). Therefore, the results reported here (including phase II data) adequately characterize the pharmacokinetics of teprotumumab.

Oncology data were included in the population PK analysis to inform model structure as only sparse PK data were collected in patients with TED. The observed PK data show that patients with TED had about 35\% lower CL compared with oncology patients, for which the mechanistic reasons are not clear. However, disease status (oncology vs TED) was not included as a covariate in the final model for a few reasons: the patient with TED is the target population in the current population PK analysis; the covariates identified in the screening covariate analysis were the same regardless of inclusion of disease status as a covariate; data fitting for the two TED studies were not improved by including disease status as a covariate; and more importantly, teprotumumab exposures in patients with TED were very similar with or without including disease status as a covariate.

Overall, teprotumumab showed no significant immunogenicity and no impact on teprotumumab pharmacokinetics. The ADA results are reliable as the phase III ADA assay had a high drug tolerance concentration of $400 \mu \mathrm{g} / \mathrm{mL}$, which is higher than the pre-dose concentrations of teprotumumab in the vast majority of patients with TED. Even for the few patients who had pre-dose concentrations above $400 \mu \mathrm{g} / \mathrm{mL}$, 
the long-term follow-up of ADA samples at weeks 36 and 72 , when teprotumumab had been washed out, was still negative. Although the ADA assay used in the phase II study did not have as high a drug tolerance concentration, the ADA samples at long-term follow-up visits (weeks 36 and 72) were also negative.

Drug-drug interactions between teprotumumab and thyroid medications (e.g., levothyroxine, propylthiouracil) commonly taken by patients with TED [10] are not expected, as teprotumumab and small-molecule drugs do not share common or overlapping CL pathways [28]. As a monoclonal antibody, teprotumumab is primarily cleared by proteolytic catabolism and broken down into peptide fragments as endogenous IgG1, thus is not subject to modulation of drug-metabolizing enzymes. Monoclonal antibodies are not expected to directly affect the hepatic, renal, or biliary elimination of small molecules. Indirect modulation of drug-metabolizing enzymes via cytokines by teprotumumab is also unlikely. Therefore, no formal drug-drug interaction studies have been conducted or are planned with teprotumumab.

\section{Conclusions}

Teprotumumab pharmacokinetics was well characterized in patients with TED. Teprotumumab showed a long elimination half-life, low systemic CL, and low volume of distribution, consistent with other IgG1 monoclonal antibodies. Efficacy was high across the exposure range of teprotumumab, and together with a well-tolerated safety profile, support the approved, and currently used, dose regimen in patients with TED.

Supplementary Information The online version contains supplementary material available at https://doi.org/10.1007/s40262-021-01003-3.

Acknowledgments We thank the patients for participating in the trials. We acknowledge the following employees of Horizon Therapeutics: Maria Kovalenko and Rui Sun, $\mathrm{PhD}$, for scientific contribution in data acquisition and analysis; Naina Barretto, $\mathrm{PhD}$, and Megan FrancisSedlak, $\mathrm{PhD}$, for writing and editorial assistance.

\section{Declarations}

Funding Study NCT00400361 was funded by Hoffman La Roche; studies NCT01868997 and NCT03461211 and the conduct of these analyses were funded by Horizon Therapeutics plc.

Conflicts of interest/Competing interest Yuying Gao and Fengyan Xu received consulting fees from Horizon Therapeutics; Yan Xin, Nivedita Bhatt, Jason Chamberlain, Saba Sile, Suzy Hammel, Robert J. Holt, Thompson, and Srini Ramanathan are employed by and own stocks in Horizon Therapeutics.
Ethics approval The trials were approved by institutional review boards of participating institutions and performed in accordance with the Declaration of Helsinki.

Consent to participate Patients provided written informed consent.

Consent for Publication Not applicable.

Availability of data and material Data will not be provided.

Code availability Not applicable.

Authors' contributions Not applicable.

Open Access This article is licensed under a Creative Commons Attribution-NonCommercial 4.0 International License, which permits any non-commercial use, sharing, adaptation, distribution and reproduction in any medium or format, as long as you give appropriate credit to the original author(s) and the source, provide a link to the Creative Commons licence, and indicate if changes were made. The images or other third party material in this article are included in the article's Creative Commons licence, unless indicated otherwise in a credit line to the material. If material is not included in the article's Creative Commons licence and your intended use is not permitted by statutory regulation or exceeds the permitted use, you will need to obtain permission directly from the copyright holder. To view a copy of this licence, visit http://creativecommons.org/licenses/by-nc/4.0/.

\section{References}

1. Smith TJ, Hegedus L. Graves' disease. N Engl J Med. 2016;375(16):1552-65. https://doi.org/10.1056/NEJMra1510030.

2. Pritchard J, Han R, Horst N, Cruikshank WW, Smith TJ. Immunoglobulin activation of $T$ cell chemoattractant expression in fibroblasts from patients with Graves' disease is mediated through the insulin-like growth factor I receptor pathway. J Immunol. 2003;170(12):6348-54. https://doi.org/10.4049/jimmunol.170. 12.6348 .

3. Douglas RS, Naik V, Hwang CJ, Afifiyan NF, Gianoukakis AG, Sand D, et al. B cells from patients with Graves' disease aberrantly express the IGF-1 receptor: implications for disease pathogenesis. J Immunol. 2008;181(8):5768-74. https://doi.org/10. 4049/jimmunol.181.8.5768.

4. Douglas RS, Gianoukakis AG, Kamat S, Smith TJ. Aberrant expression of the insulin-like growth factor- 1 receptor by $\mathrm{T}$ cells from patients with Graves' disease may carry functional consequences for disease pathogenesis. J Immunol. 2007;178(5):32817. https://doi.org/10.4049/jimmunol.178.5.3281.

5. Tsui S, Naik V, Hoa N, Hwang CJ, Afifiyan NF, Sinha Hikim A, et al. Evidence for an association between thyroid-stimulating hormone and insulin-like growth factor 1 receptors: a tale of two antigens implicated in Graves' disease. J Immunol. 2008;181(6):4397-405. https://doi.org/10.4049/jimmunol.181.6. 4397.

6. Smith TJ, Janssen J. Insulin-like growth factor-I receptor and thyroid-associated ophthalmopathy. Endocr Rev. 2019;40(1):236-67. https://doi.org/10.1210/er.2018-00066.

7. Smith TJ. Novel aspects of orbital fibroblast pathology. J Endocrinol Invest. 2004;27(3):246-53. https://doi.org/10.1007/BF033 45273.

8. Krieger CC, Boutin A, Jang D, Morgan SJ, Banga JP, Kahaly GJ, et al. Arrestin-beta-1 physically scaffolds TSH and IGF1 receptors 
to enable crosstalk. Endocrinology. 2019;160(6):1468-79. https:// doi.org/10.1210/en.2019-00055.

9. Smith TJ, Kahaly GJ, Ezra DG, Fleming JC, Dailey RA, Tang RA, et al. Teprotumumab for thyroid-associated ophthalmopathy. N Engl J Med. 2017;376(18):1748-61. https://doi.org/10.1056/ NEJMoa1614949.

10. Douglas RS, Kahaly GJ, Patel A, Sile S, Thompson EHZ, Perdok $\mathrm{R}$, et al. Teprotumumab for the treatment of active thyroid eye disease. N Engl J Med. 2020;382(4):341-52. https://doi.org/10. 1056/NEJMoa1910434.

11. Tepezza [prescribing information]. 2020. https://www.accessdata. fda.gov/drugsatfda_docs/label/2020/761143s000lbl.pdf. Accessed 20 Feb 2021

12. Kurzrock R, Patnaik A, Aisner J, Warren T, Leong S, Benjamin R, et al. A phase I study of weekly R1507, a human monoclonal antibody insulin-like growth factor-I receptor antagonist, in patients with advanced solid tumors. Clin Cancer Res. 2010;16(8):245865. https://doi.org/10.1158/1078-0432.CCR-09-3220.

13. Rodon JPA, Stein M, Tolcher A, Ng C, Dias M, Kurman C, et al. A phase I study of q3W R1507, a human monoclonal antibody IGF-1R antagonist in patients with advanced cancer. J Clin Oncol. 2007;25(18 Suppl. 3590).

14. Yang BB, Lum P, Chen A, Arends R, Roskos L, Smith B, et al. Pharmacokinetic and pharmacodynamic perspectives on the clinical drug development of panitumumab. Clin Pharmacokinet. 2010;49(11):729-40. https://doi.org/10.2165/11535970-00000 0000-00000.

15. Beal SL, Boeckmann AJ, Sheiner LB. NONMEM user's guide, Part I-VII. San Francisco (CA): University of California at San Francisco; 1988. 1992.

16. Cockcroft DW, Gault MH. Prediction of creatinine clearance from serum creatinine. Nephron. 1976;16(1):31-41. https://doi.org/10. 1159/000180580.

17. Bergstrand M, Hooker AC, Wallin JE, Karlsson MO. Prediction-corrected visual predictive checks for diagnosing nonlinear mixed-effects models. AAPS J. 2011;13(2):143-51. https://doi. org/10.1208/s12248-011-9255-z.

18. Harling K, Uekcert S, Hooker A. NPC/VPC userguide and technical description. PsN 3.4.2. http://psn.sourceforge.net/pdfdocs/ npc_vpc_userguide.pdf. Accessed 2013.

19. Ette EI. Stability and performance of a population pharmacokinetic model. J Clin Pharmacol. 1997;37(6):486-95. https://doi. org/10.1002/j.1552-4604.1997.tb04326.x.

20. Bootstrap user guide. 2011.

21. Deng R, Jin F, Prabhu S, Iyer S. Monoclonal antibodies: what are the pharmacokinetic and pharmacodynamic considerations for drug development? Expert Opin Drug Metab Toxicol. 2012;8(2):141-60. https://doi.org/10.1517/17425255.2012. 643868 .

22. Ryman JT, Meibohm B. Pharmacokinetics of monoclonal antibodies. CPT Pharmacometrics Syst Pharmacol. 2017;6(9):576-88. https://doi.org/10.1002/psp4.12224.

23. Dirks NL, Meibohm B. Population pharmacokinetics of therapeutic monoclonal antibodies. Clin Pharmacokinet. 2010;49(10):63359. https://doi.org/10.2165/11535960-000000000-00000.

24. Mager DE. Target-mediated drug disposition and dynamics. Biochem Pharmacol. 2006;72(1):1-10. https://doi.org/10.1016/j.bcp. 2005.12.041.

25. Perros P, Neoh C, Dickinson J. Thyroid eye disease. BMJ. 2009;338:b560.

26. Kahaly GJ, Smith TJ, Holt RJ, Sile S, Douglas RS. Effect of teprotumumab on proptosis reduction across various demographic subgroups (abstract). J Endo Soc. 2020;4(Suppl. 1):A559-60.

27. Scientific T. Protein storage and stability (tech tip \#43).

28. Zhou H, Mascelli MA. Mechanisms of monoclonal antibody-drug interactions. Annu Rev Pharmacol Toxicol. 2011;51:359-72. https://doi.org/10.1146/annurev-pharmtox-010510-100510. 